\title{
CIRCUMSTELLAR MOLECULAR ENVELOPES
}

\author{
G. A. FULLER
}

Physics Department, UMIST

P.O. Box 88, Manchester, M60 1QD, UK

\author{
AND \\ E. F. LADD \\ Five College Radio Astronomy Observatory \\ University of Massachusetts, Amherst, MA 01003, USA \\ and \\ Department of Physics, Bucknell University \\ Lewisburg, PA 17837, USA
}

\begin{abstract}
The results of $\mathrm{C}^{17} \mathrm{O}$ and $\mathrm{C}^{18} \mathrm{O}$ observations of low luminosity young stars are discussed and the derived properties of the circumstellar molecular envelopes described. Much of the circumstellar material is quiescent and cold. The youngest sources have envelopes which contain up to $\sim 0.3 \mathrm{M}_{\odot}$ of material within $3000-4000 \mathrm{AU}$ of the central star. From a survey of sources in Taurus, both the timescale and mechanism of clearing of the circumstellar region are investigated. Neither the observed outflows nor standard infall models alone are capable of clearing the circumstellar regions quickly enough. Maps of the circumstellar material around two very young sources suggest that the stars form from flattened configurations of material.
\end{abstract}

\section{Introduction}

The structure and dynamics of circumstellar material play a large role in determining how a protostar forms and evolves. The study of the circumstellar regions of protostars therefore provides key details for understanding how stars and circumstellar disks form and evolve.

The millimetre and submillimetre continuum emission from young stars clearly shows the presence of significant amounts of circumstellar mate- 

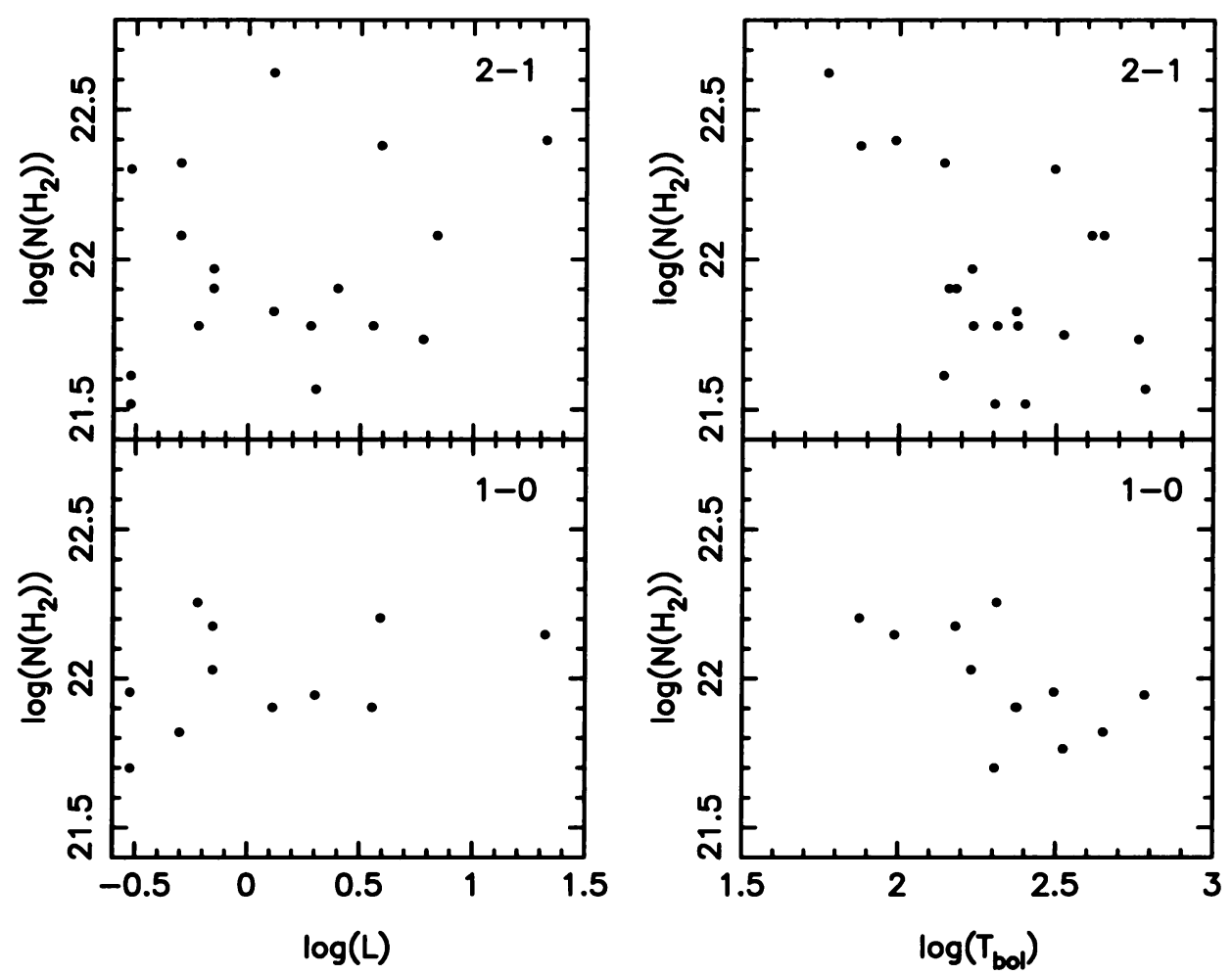

Figure 1. Column density derived from $\mathrm{C}^{18} \mathrm{O}$ and $\mathrm{C}^{17} \mathrm{O}$ observations versus source luminosity (left) and bolometric temperature (right). The upper figures show the results derived from the $J=2 \rightarrow 1$ transitions and the lower figures show the results from the $\mathrm{J}=1 \rightarrow 0$ transitions.

rial associated with these stars (Ladd et al. 1991a,b) and modeling of this emission has provided constraints on the density and temperature of circumstellar material (e.g. Terebey et al., 1993; André \& Montmerle, 1994). However, there has been little systematic work on molecular line tracers of this material. Molecular line probes of these regions provide a different view of the circumstellar material, one which is less biased by the temperature structure of the regions. The most significant advantage of molecular probes over dust is of course that the velocity and line width of spectral lines are direct probes of the dynamics of the circumstellar material.

We have examined the circumstellar regions of young stars in molecular lines using two complementary approaches. First, we have completed a single-beam survey of sources in Taurus using the $\mathrm{J}=1 \rightarrow 0$ and $\mathrm{J}=2 \rightarrow 1$ transitions of $\mathrm{C}^{18} \mathrm{O}$ and $\mathrm{C}^{17} \mathrm{O}$ using FCRAO and CSO. In parallel with this survey, we are mapping selected sources in these lines and the $\mathrm{J}=3 \rightarrow 2$ 


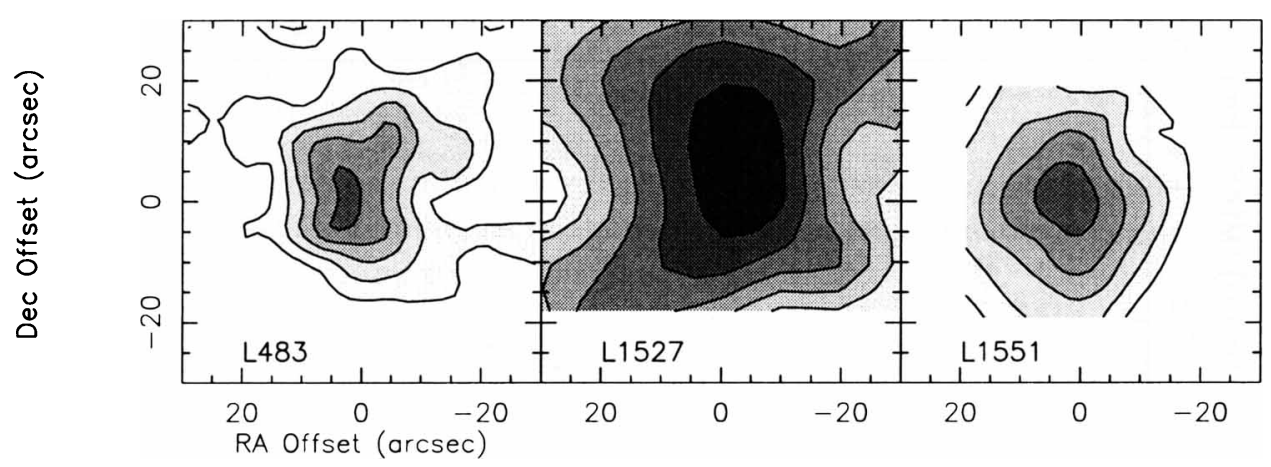

Figure 2. Maps of the dense circumstellar material around three young sources. Left: Map of the integrated intensity of the $\mathrm{C}^{18} \mathrm{O} \mathrm{J}=3 \rightarrow 2$ around the embedded source in L483. Centre: Integrated $\mathrm{C}^{17} \mathrm{O} \mathrm{J}=2 \rightarrow 1$ emission towards the the embedded source in L1527 (Fuller et al., 1996). Right: Integrated $\mathrm{C}^{17} \mathrm{O} \mathrm{J}=2 \rightarrow 1$ emission towards the embedded source in L1551 (Fuller et al., 1995).

transitions of the same species using FCRAO and JCMT.

\section{Rare Species of CO As Probes}

Our choice of $\mathrm{C}^{17} \mathrm{O}$ and $\mathrm{C}^{18} \mathrm{O}$ as probes of the circumstellar material is based on the fact that these isotopomers of carbon monoxide typically have low optical depth toward young stellar objects, that because of their low dipole moment the low-J energy levels are thermalized in these regions, and that the abundances of these isotopomers appear to be little affected by chemical or dynamical processes within the cloud. $\mathrm{C}^{17} \mathrm{O}$ is a factor of $\sim 10^{3}$ less abundant than the most common $\mathrm{CO}$ isotope, ${ }^{12} \mathrm{CO}$, and its lines are optically thin for beam-averaged column densities in excess of $10^{23} \mathrm{~cm}^{-2}$. By observing the low optical depth lines of two isotopomers, we can calculate the optical depth, column density, and excitation temperature of the emitting material.

Figure 1 shows that these CO isotopomers trace the circumstellar material around young sources. The hydrogen column densities derived from observations of the $\mathrm{J}=1 \rightarrow 0$ and the $\mathrm{J}=2 \rightarrow 1$ lines are not correlated with the source luminosity but are correlated with the source bolometric temperature, $T_{\text {bol }}$ (Myers \& Ladd, 1993). As a characteristic of the spectral energy distribution of an embedded source, $T_{\mathrm{bol}}$ measures the amount of circumstellar dust around a young star; sources with higher values of $T_{\mathrm{bol}}$ have less circumstellar dust. Since the column density derived from the CO observations decreases with increasing $T_{\mathrm{bol}}$, the $\mathrm{CO}$ is tracing the same circumstellar material as the dust measured by $T_{\text {bol }}$. The correlation also 


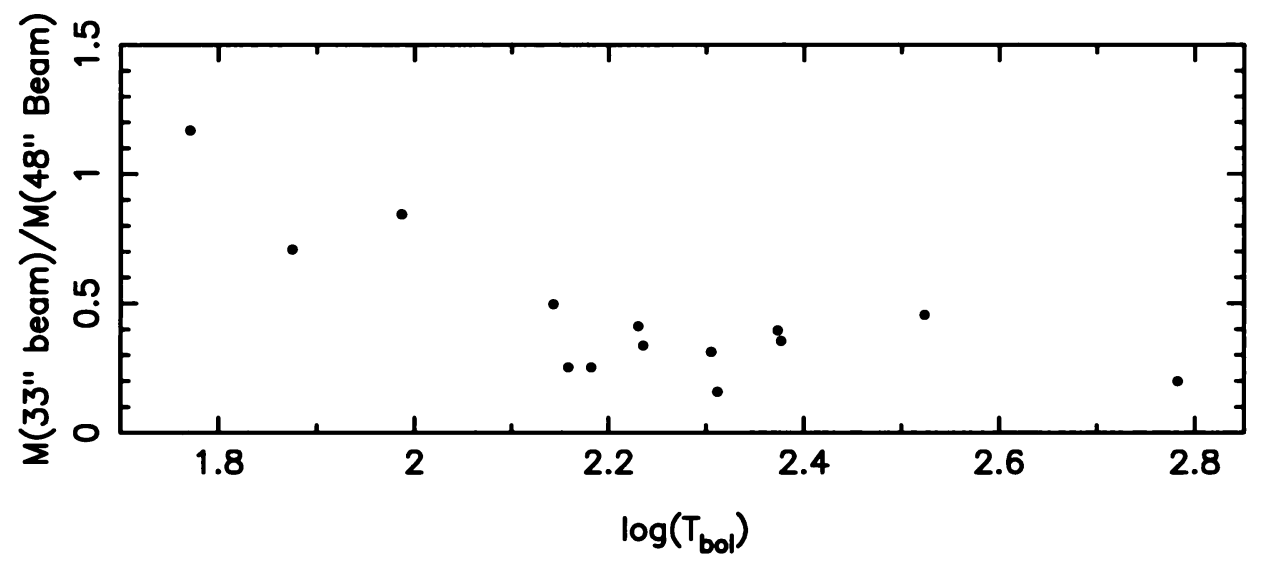

Figure 3. Ratio of beam mass derived from the $\mathrm{J}=2 \rightarrow 1$ observations to that derived from the $\mathrm{J}=1 \rightarrow 0$ observations versus source bolometric temperature. Two sources, L1536 and IRAS04181B, are not shown on this figure as the column densities derived towards these sources are thought to be unreliable (Ladd et al., 1997).

shows that the decrease in gas column density is not due to depletion of the gas phase molecules onto dust grains.

It has been argued that the $1.3 \mathrm{~mm}$ dust continuum emission from young stellar objects is directly proportional to the mass of the circumstellar envelope (André et al., 1993; Saraceno et al., 1996). However, the $1.3 \mathrm{~mm}$ flux of both outflow sources and HH exciting sources is strongly correlated with the source luminosity (Saraceno et al., 1996; Reipurth et al., 1993). This correlation suggests that the $1.3 \mathrm{~mm}$ emission is not a function of mass alone but is determined by a combination of both the mass and temperature of the circumstellar material. The absence of such a correlation in the CO data shows the column density and mass results (and the observed line integrated intensities) to be relatively insensitive to temperature.

The most direct evidence that $\mathrm{C}^{18} \mathrm{O}$ and $\mathrm{C}^{17} \mathrm{O}$ trace the circumstellar material comes from the sources which have been mapped. Three of these sources are shown in Figure 2. The maps show that $\mathrm{C}^{17} \mathrm{O} / \mathrm{C}^{18} \mathrm{O}$ emission is centred on the young stars and have a typical radial extent of $\sim 3000 \mathrm{AU}$.

\section{Mass of Circumstellar Material}

Both the survey and maps show that the circumstellar regions contain a substantial mass of material, with the youngest sources having the most circumstellar material. The Taurus survey shows that within about $3400 \mathrm{AU}$ of the central stars the mass of the circumstellar material ranges from about $0.3 \mathrm{M}_{\odot}$ for the youngest sources to $0.02 \mathrm{M}_{\odot}$ for the older sources. Similarly 
the objects which have been mapped, which are the most embedded sources, show 0.2 to $0.5 \mathrm{M}_{\odot}$ within a region 3000 to $4000 \mathrm{AU}$ in radius around the sources.

These masses are significantly higher than derived from other molecular line observations. An analysis of the CS emission from a sample containing some of the same young sources in Taurus as observed in CO, found circumstellar masses ranging from $0.003 \mathrm{M}_{\odot}$ to $0.015 \mathrm{M}_{\odot}$ (Moriarty-Schieven et al., 1995). The CS emission also has considerably larger velocity dispersion and higher temperature than the $\mathrm{C}^{17} \mathrm{O}$. This suggests that the CS emission is a relatively poor tracer of the quiescent circumstellar material and may instead be associated with the outflows from the young stars. Maps of some sources show that there is considerable CS emission associated with the outflows, for example in NGC 1333 IRAS2 (Sandell et al., 1994). Also, towards L1527 a high resolution map of CS $\mathrm{J}=2 \rightarrow 1$ by Ohashi et al. (1996) shows the emission peaking $20^{\prime \prime}$ from the location of the embedded source whereas the $\mathrm{C}^{17} \mathrm{O} \mathrm{J}=2 \rightarrow 1$ emission is centred on the source (Figure 2).

The $\mathrm{J}=1 \rightarrow 0$ and $\mathrm{J}=2 \rightarrow 1$ data have been analysed independently and therefore produce independent estimates of the column density towards the sources. Figure 3 shows the ratio of the mass within the two beams. Since the beams have different FWHM, $48^{\prime \prime}$ for the $\mathrm{J}=1 \rightarrow 0$ observations and $33^{\prime \prime}$ for the $\mathrm{J}=2 \rightarrow 1$ observations, this ratio depends on the density profile of the material around each source. If the material is uniformly distributed around a source the ratio of mass in the smaller $\mathrm{J}=2 \rightarrow 1$ beam to the larger $\mathrm{J}=1 \rightarrow 0$ would just scale as the beam area and so be 0.47 . On the other hand, if the material is concentrated near the star, the ratio should increase, and in the limit approach unity, as the larger beam would contain only a slightly larger mass of material than the smaller beam. Figure 3 shows that there is a inverse correlation between this ratio and $T_{\text {bol }}$.

The younger sources have higher values of this ratio and are consistent with their circumstellar material being strongly centrally condensed. The older, high $T_{\text {bol }}$ sources have low values of the ratio and are consistent with uniform distributions of material. This change from centrally condensed to consistent with a uniform distribution of circumstellar material suggests that as material is removed from the circumstellar regions, it is removed first, and most significantly, from the region closest to the forming star.

\section{The Temperature of the Circumstellar Material}

Both the $\mathrm{J}=1 \rightarrow 0$ and $\mathrm{J}=2 \rightarrow 1$ survey data indicate excitation temperatures of 5 to $14 \mathrm{~K}$ for the circumstellar material. These values are consistent with the 7 to $15 \mathrm{~K}$ found from the $\mathrm{J}=2 \rightarrow 1$ maps. The ratio of $\mathrm{J}=2 \rightarrow 1$ to $\mathrm{J}=3 \rightarrow 2$ emission suggests somewhat higher excitation temperature for 
TABLE 1. Fits to lines towards

L1527

Line $\quad \mathrm{dv}\left(\mathrm{kms}^{-1}\right)$

Comp. 1 Comp. 2

\begin{tabular}{ccc}
\hline $\mathrm{C}^{18} \mathrm{O} 1-0$ & 0.47 & 1.7 \\
$\mathrm{C}^{17} \mathrm{O} 1-0$ & 0.41 & 1.2 \\
$\mathrm{C}^{18} \mathrm{O} 2-1$ & 0.57 & 1.87 \\
$\mathrm{C}^{17} \mathrm{O} 2-1$ & 0.44 & $\ldots$ \\
$\mathrm{C}^{18} \mathrm{O} 3-2$ & 0.54 & 2.01 \\
$\mathrm{C}^{17} \mathrm{O} 3-2$ & 0.50 & $\ldots$
\end{tabular}

some sources; this result is not unexpected as the $\mathrm{J}=3 \rightarrow 2$ emission is likely to contain a contribution from warmer material. The comparison of these two lines is, however, more uncertain due to the different beam sizes of the observations. These temperatures are lower than has been estimated from either CS J=5 $\rightarrow 4$ (Moriarty-Schieven et al., 1995) or the dust continuum emission, even for Class 0 sources which have spectral energy distributions which are well matched by single temperature models (Moriarty-Schieven et al., 1994; André et al., 1993).

The excitation temperatures are in reasonable agreement with the models of Ceccarelli, Hollenbach and Tielens (1996). Scaling the results from their $20 \mathrm{~L}_{\odot}$ model to $1 \mathrm{~L}_{\odot}$ (which is typical of the Taurus sources), yields a temperature of $9.5 \mathrm{~K}$ at a radius of $4.2 \times 10^{16} \mathrm{~cm}(2800 \mathrm{AU})$ for the forming star.

\section{The Velocity Dispersion of the Circumstellar Material}

Most of the sources show a narrow line width component $\left(\sim 0.5-0.7 \mathrm{~km} \mathrm{~s}^{-1}\right)$, indicating that much of the circumstellar material is relatively quiescent. This narrow velocity component is not seen in CS $\mathrm{J}=5 \rightarrow 4$ (MoriartySchieven et al., 1995).

Some sources also show a second broader component which is more apparent in $\mathrm{C}^{18} \mathrm{O}$ than in $\mathrm{C}^{17} \mathrm{O}$ and in the higher $\mathrm{J}$ transitions than in the lower $\mathrm{J}$ transitions, suggesting that the broad component has lower optical depth and is warmer than the narrow component. An example of Gaussian fits to the line profiles towards the embedded source in L1527 is given in Table 1. Notice the consistency of the linewidth of the narrow component and that for a given transition the $\mathrm{C}^{17} \mathrm{O}$ linewidth is narrower, consistent with the $\mathrm{C}^{18} \mathrm{O}$ line being more optically thick than the $\mathrm{C}^{17} \mathrm{O}$ line. 


\section{Variation of Column Density with $T_{\text {bol }}$}

It has been suggested that sources may have low values of $T_{\mathrm{bol}}$, not because they have large amounts of circumstellar material, but because they are viewed edge-on. The column density- $T_{\text {bol }}$ correlation shown in Figure 1 shows that this is not the case. The derived column densities are independent of inclination angle and the low $T_{\text {bol }}$ sources have the highest column densities of material, and the most massive circumstellar envelopes.

$T_{\text {bol }}$ is an age ordering quantity and $T_{\text {bol }}$ can be correlated with age estimates for the sources (Chen et al., 1995; Ladd et al., 1997). Using this correlation together with the column density- $T_{\text {bol }}$ correlation it is possible to investigate how the amount of circumstellar material decreases with source age.

The column density is found to vary with source age, $t_{\mathrm{yrs}}$, as

$$
\begin{array}{ll}
48^{\prime \prime} \text { beam } \log \left(N_{\mathrm{H}_{2}}\right)=2.9-0.42 \log \left(t_{\mathrm{yrs}}\right) \pm 0.29 \\
33^{\prime \prime} \text { beam } \log \left(N_{\mathrm{H}_{2}}\right)=4.3-0.74 \log \left(t_{\mathrm{yrs}}\right) \pm 0.45
\end{array}
$$

for the $\mathrm{J}=1 \rightarrow 0$ and $\mathrm{J}=2 \rightarrow 1$ data respectively. These relations can be converted to the rate at which mass is lost from the respective beams by multiplying the column density by the respective beam size and differentiating with respect to the source age. This leads to beam mass loss rates of

$$
\begin{array}{ll}
48^{\prime \prime} \text { beam } \log \left(\dot{\mathrm{M}}_{\text {beam }}\right)=0.7-1.42 \log \left(t_{\mathrm{yrs}}\right) \pm 0.41 \\
33^{\prime \prime} \text { beam } \log \left(\dot{\mathrm{M}}_{\text {beam }}\right)=2.0-1.74 \log \left(t_{\mathrm{yrs}}\right) \pm 0.60
\end{array}
$$

These show that both beam mass loss rates are decreasing functions of source age. For the youngest source, L1527, $\dot{\mathrm{M}}_{\text {beam }}>10^{-5} \mathrm{M}_{\odot} \mathrm{yr}^{-1}$ while for older sources such as HL Tau, $\dot{\mathrm{M}}_{\text {beam }}<10^{-7} \mathrm{M}_{\odot} \mathrm{yr}^{-1}$.

There are two ways in which the mass within the beam could decrease. Material could be moved out of the beam by the outflows from the star, alternatively material could collapse to a compact optically thick region to which the observations are insensitive.

\subsection{OUTFLOW}

The role of outflows in removing material from our beam can be assessed using the mass loss rates derived from the observed bipolar outflows associated with the sources. Of the sources observed into the $\mathrm{J}=2 \rightarrow 1$ transitions, 11 have outflows detected in $\mathrm{CO}$.

In order to determine whether the outflows could remove the circumstellar material at the rate required by the $\mathrm{C}^{17} \mathrm{O} / \mathrm{C}^{18} \mathrm{O}$ observations, it is important to understand the origin of the $\mathrm{CO}$ in the observed outflows. If all the $\mathrm{CO}$ in the bipolar outflows is material that has been entrained 


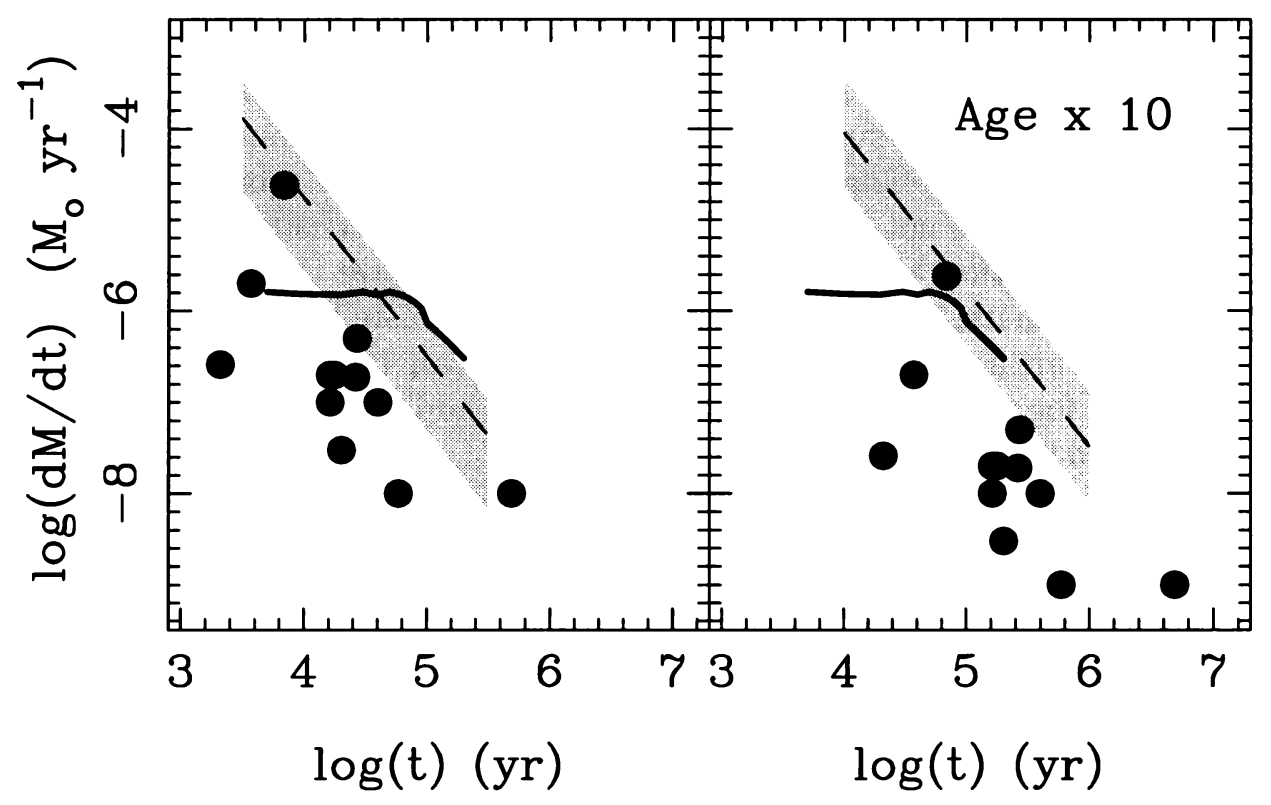

Figure 4. Mass loss rates derived from the $\mathrm{J}=2 \rightarrow 1$ observations versus source age. In each panel the dashed line and gray region indicate the best fit beam mass loss rate and the estimated uncertainty from the $\mathrm{J}=2 \rightarrow 1 \mathrm{C}^{17} \mathrm{O} / \mathrm{C}^{18} \mathrm{O}$ data. The points indicate the mass loss rates due to the outflows from the sources. The solid line is the beam mass loss rate for a collapsing singular isothermal sphere at $10 \mathrm{~K}$. The panel to the left shows the results for using the $T_{\mathrm{bol}}$ - source age correlation discussed in the text. The other panel shows the results if the sources are assumed to be 10 times older than implied by the $T_{\text {bol }}$ correlation.

from the circumstellar regions around the young stars, then the mass loss rate from the circumstellar region is just equal to the $\mathrm{CO}$ outflow mass loss rate. If, however, the $\mathrm{CO}$ is only entrained at large distances from the central star, outside the beams used to make the $\mathrm{C}^{17} \mathrm{O} / \mathrm{C}^{18} \mathrm{O}$ observations, the circumstellar mass loss rate is equal to the stellar wind mass loss rate. This is smaller than the observed CO outflow mass loss rate by the ratio of the outflow speed to the wind speed, a factor typically assumed to be 0.1 .

The presence of shocks and $\mathrm{HH}$ objects at large distances from the central sources together with high angular resolution observations of outflows within a few arcseconds of young stars (e.g. Chandler et al. 1996) suggest that entrainment takes place over much of the length of an outflow. Therefore in comparing the outflow mass loss rates with the $\mathrm{C}^{17} \mathrm{O} / \mathrm{C}^{18} \mathrm{O}$ data we have assumed that the circumstellar mass loss rate is one third of the outflow mass loss rate.

The outflow mass loss rates are compared with the observed beam mass 
loss rates derived from the $\mathrm{J}=2 \rightarrow 1 \mathrm{C}^{17} \mathrm{O} / \mathrm{C}^{18} \mathrm{O}$ observations in Figure 4. Except for one source, L1551 IRS5 (Fuller et al. 1995), the outflow mass loss rates fall below the $\mathrm{C}^{17} \mathrm{O} / \mathrm{C}^{18} \mathrm{O}$ rates, in many cases by an order of magnitude. The outflows therefore do not appear capable of removing the circumstellar material sufficiently quickly to match the observed decline in the mass of circumstellar material. Although only the results from the $\mathrm{J}=2 \rightarrow 1$ observations are shown in Figure 4, analysis of the $\mathrm{J}=1 \rightarrow 0$ results lead to the same conclusions about role of outflows (and infall) in clearing the circumstellar regions (Ladd et al., 1997).

\subsection{INFALL}

In the standard singular isothermal sphere (SIS) model for the collapse of a dense core, the central regions of the core collapse first and an expansion wave marking the onset of collapse propagates outwards at the local sound speed. The mass accretion rate during the collapse is constant and given by

$$
\dot{M}=0.975 \frac{a^{3}}{G}
$$

where $a$ is the isothermal sound speed (Shu 1977). For a gas temperature of $10 \mathrm{~K}$, the mass accretion rate is therefore $1.6 \times 10^{-6} \mathrm{M}_{\odot} \mathrm{yr}^{-1}$. The rate of loss of mass within the beam used for the $\mathrm{J}=2 \rightarrow 1$ observations due to a 10K SIS infall is shown as the solid curve in Figure 4. The left panel of the figure shows that at early times the mass loss rate from the beam is constant at $1.6 \times 10^{-6} \mathrm{M}_{\odot} \mathrm{yr}^{-1}$ as material collapses into the central region where it becomes optically thick and invisible to our observations. At a time $5.8 \times 10^{4}$ years after the start of the collapse, the expansion wave radius is equal to the radius of the $\mathrm{J}=2 \rightarrow 1$ beam. After this time the beam mass loss rate decreases since once the expansion wave radius is larger than the beam, material initially outside the beam starts to infall and compensate for material which is collapsing at centre of the core and becoming undetectable.

The beam mass loss rate for the 10K SIS model is significantly smaller than the rate derived from the observations for the youngest sources. But for sources older than about $4 \times 10^{5}\left(10^{4.6}\right)$ years the SIS beam mass loss rate exceeds that observed. For the older sources this infall model could therefore account for the observed decrease of circumstellar material. A SIS model with a temperature of $20 \mathrm{~K}$, for which the sound speed is consistent with the observed line widths of the $\mathrm{C}^{18} \mathrm{O}$, has a higher beam mass loss rate than the $10 \mathrm{~K}$ model at all times. Such a model has a mass loss rate equal to the observed beam mass loss rate for slightly younger sources than 
the $10 \mathrm{~K}$ model, but still can not match the very high mass loss rate for the youngest sources.

Perhaps the largest uncertainty in comparing the infall model and the observations is the ages of the sources. The effect of artificially increasing the age of all the sources by a factor of 10 is shown in the right hand panel of Figure 4. In this figure all the sources are assumed to be 10 times older than in the other panel. The best fit to the beam mass loss rate is shown as the dashed line and its uncertainty is indicated by the shaded area. The solid points indicate the outflow mass loss rates. The assumed greater age of the sources has moved these points down by an order of magnitude. This occurs because these values have been derived using the outflow dynamical age as an estimate of the source age and therefore would decrease if the sources were in fact older than their dynamical ages suggest (Parker et al., 1991; Padman \& Richer, 1994; Masson \& Chernin, 1993). Increasing the source ages therefore does not affect the relative locations of the outflow mass loss rates and observed beam mass loss rates.

The solid line shows again the 10K SIS model. Since the location of this curve is fixed by the properties of the infall, its location is unaffected by the larger age of the sources. However, the greater age of the sources has moved the best fit beam mass loss rate further from the SIS model. For the assumed factor of ten increase in source ages, this $10 \mathrm{~K}$ SIS model never exceeds the best fit beam mass loss rate for the sources.

\subsection{OUTFLOW AND INFALL?}

The discussion above shows that the outflows from the sources are unlikely to alone account for the observed clearing of the circumstellar molecular cores. On the other hand, SIS infall models can not match the observations for the youngest sources. This suggests two possible conclusions. The first is that at early times the sources have mass accretion rates much higher than in the standard SIS model. Infall models based on models of cores other than as singular isothermal spheres such as Bonner-Ebert spheres predict higher initial (and time variable) mass accretion rates (Foster \& Chevalier, 1993; Henriksen et al., 1997) and Bontemps et al. (1996) have invoked high mass accretion rates for the youngest embedded sources to explain the more energetic outflows from these sources.

The alternative possibility for the observed beam mass loss rates is that early in the evolution of young sources the stellar winds are very efficiently coupled to the dense circumstellar material. The outflows are therefore responsible for clearing the circumstellar material for the youngest sources but later in their evolution, at times greater than about $4 \times 10^{4}$ years, infall becomes the dominant process by which the remaining circumstellar 
material is cleared. It might indeed be expected that the stellar winds are much better coupled to the dense circumstellar material if they initially have broken out of the dense circumstellar environment, but later escape the circumstellar regions predominantly through these cleared paths.

\section{The Structure of the Circumstellar Environment}

Maps showing the distribution of the material traced by $\mathrm{C}^{17} \mathrm{O}$ and $\mathrm{C}^{18} \mathrm{O}$ around three young stars are shown in Figure 2. Of these three sources, the oldest as measured by its bolometric temperature of $96 \mathrm{~K}$, is L1551 IRS-5. Towards this source the $\mathrm{C}^{17} \mathrm{O}$ emission traces two components of circumstellar material: a cross of material with the arms of the cross aligned in the cardinal directions and a more extended cooler, more quiescent component. Both these components are also visible in the submillimetre continuum emission. The continuum and line emission give somewhat different relative masses of these two components with the line emission suggesting that not more than half the total mass is in the cross component whereas the continuum emission is consistent with a much smaller fraction of the total mass in a hot cross component. The origin of this cross structure has been discussed in detail by Ladd et al. (1995) and Fuller et al. (1995) who conclude that it is tracing the warm region of interaction between the outflow from the source and the walls of the outflow cavity.

The two younger sources shown in Figure 2, L483 and L1527 which have bolometric temperatures of $46 \mathrm{~K}$ and $59 \mathrm{~K}$ respectively, both show similar circumstellar structures. The outflows from both sources are aligned close to east-west and in each case the rare isotopes of CO show a $2000-3000$ AU radius structure elongated perpendicular to the outflow axis. These structures have masses of $\sim 0.5 \mathrm{M}_{\odot}$ and $\sim 0.3 \mathrm{M}_{\odot}$ for L483 and L1527 respectively.

Flattened structures of this physical size around other sources, most notably HL Tau (Hayashi et al., 1993), have been interpreted as examples of pseudo-disks resulting from the collapse of a dense core (Galli \& Shu, 1993a,b). However, there is a problem with describing the structures around the sources in L483 and L1527 as pseudo-disks. The pseudo-disks of Galli \& Shu result from the collapse of an initially spherical dense core and only produce flattened structures as large and massive as seen in L1527 and L483 at times greater than $10^{5}$ years. This is at least an order of magnitude older than the estimated ages of these sources, which for L1527 is $\sim 5000$ years.

It seems unlikely that these structures are the remnants of initially spherical distributions as this would require the outflows to have cleared material from a very large solid angle around the sources in a very short time. Perhaps the simplest interpretation of these flattened structures is 
that they predate the formation of the central star, so that the young stars have formed from flattened distributions of dense gas. This would be consistent with the modeling of reflection nebulae associated with embedded sources which are best explained if the stars form from such flattened structures (Hartmann et al., 1996). The formation, structure and evolution of such flattened initial configurations has recently been investigated by $\mathrm{Li} \&$ Shu $(1996 ; 1997)$. If flattened structures are the initial configurations out of which stars form, all young sources should be surrounded by such structures and there should be little correlation between the properties of the structures and the properties of the outflows from the stars. Observations of other extremely young sources should allow us to better understand the properties and origins of these structures.

\section{References}

André, P., and Montmerle, T. 1994, ApJ, 420, 837

André, P., Ward-Thompson, D., and Barsony, M. 1993, ApJ, 406, 122

Bontemps, S., Andre, P., Terebey, S., and Cabrit, S. 1996, AA, 311, 858

Ceccarelli, C., Haas, M. R., Hollenbach, D. J., and Rudolph, A., L. 1997, ApJ, 476, 771

Chandler, C. J., Terebey, S., Barsony, M., Moore, T. J. T., and Gautier, T. N. 1996, ApJ, 471,308

Chen, H., Myers, P. C., Ladd, E. F., and Wood, D. O. S. 1995, ApJ, 445, 377

Foster, P. N., and Chevalier, R. A. 1993, ApJ, 416, 303

Fuller, G. A., Ladd, E. F., and Hodapp, K. -W. 1996, ApJL, 463, L97

Fuller, G. A., Ladd, E. F., Padman, R., Myers, P. C., and Adams, F. C. 1995, ApJ, 454, 862

Galli, D., and Shu, F. H. 1993a, ApJ, 417, 220

Galli, D., and Shu, F. H. 1993b, ApJ, 417, 243

Hartmann, L., Calvet, N., and Boss, A, 1996, ApJ, 464, 387

Hayashi, M., Ohashi, N., and Miyama, S. M. 1993, ApJ, 418,71

Henriksen, R., André, P., and Bontemps, S. 1997, AA, in press

Ladd, E. F., Fuller, G. A., and Deane, J. R. 1997, ApJ in press

Ladd, E. F., Fuller, G. A., Padman, R., Myers, P. C., and Adams, F. C. 1995, ApJ, 439, 771

Li, Z-Y, and Shu, F. H. 1996, ApJ 472, 211

$\mathrm{Li}, \mathrm{Z}-\mathrm{Y}$, and Shu, F. H. 1997, ApJ 475, 237

Masson, C., R., and Chernin, L. M. 1993, ApJ, 414, 230

Moriarty-Schieven, G. H., Wannier, P. G., Mangum, J. G., Tamura, M., and Olmsted, V. K. 1995, ApJ, 455, 190

Moriarty-Schieven, G. H., Wannier, P. G., Tamura, M., and Keene, J. 1994, ApJ, 400, 260

Myers, P. C., and Ladd, E. F. 1993, ApJL, 413, 47

Ohashi, N., And Hayashi, M., Kawabe, R., And Ishiguro, M. 1996, ApJ, 466, 317

Padman, R., and Richer, J. S. 1994, APSS, 216, 129

Parker, N. D., Padman, R., and Scott, P. F. 1991, MNRAS, 252, 442

Reipurth, B., Chini, R., Krugel, E., Kreysa, E., and Sievers, A. 1993, AA, 273, 221

Sandell, G., Knee, L. B. G., Aspin, C., Robson, I. E., and Russell, A. P. G. 1994, AA, 285,1

Saraceno, P., André, P., Ceccarelli, C., Griffin, M., and Molinari, S. 1996, AA, 309, 827

Shu, F. H. 1977, ApJ, 214, 488

Terebey, S., Chandler, C. J., and André, P. 1993, ApJ, 414, 759 